MS25 Quasicrystal and

approximant: structure and properties

\author{
Chairs: Cesar Pay Gomez, Emilie Gaudry
}

\section{MS25-01 Computational Self-Assembly of Complex Crystals}

Julia Dshemuchadse ${ }^{1}$, Michael Engel ${ }^{1,2}$, Pablo F. Damasceno ${ }^{3,4}$, Carolyn L. Phillips ${ }^{4,5}$, Sharon C. Glotzer ${ }^{1,4,6,7}$

1. Department of Chemical Engineering, University of Michigan, Ann Arbor, Michigan, USA

2. Department of Chemical and Biological Engineering, Friedrich-Alexander-Universität Erlangen-Nürnberg, Erlangen, Germany

3. Department of Cellular and Molecular Pharmacology, University of California, San Francisco, California, USA

4. Applied Physics Program, University of Michigan, Ann Arbor, Michigan, USA

5. Argonne National Laboratory, Argonne, Illinois, USA

6. Department of Materials Science and Engineering, University of Michigan, Ann Arbor, Michigan, USA

7. Biointerfaces Institute, University of Michigan, Ann Arbor, Michigan, USA

email:djulia@umich.edu

Complex structures can be found in all classes of materials and across multiple length scales. We seek to understand why and how these geometries arise - both aperiodic ones [1], as well as periodic structures with hundreds or thousands of atoms or particles per unit cell (e.g., [2]). We computationally study particles interacting via isotropic pair potentials and analyze the structures into which they self-assemble. We simulate a wide range of systems using the highly parallel molecular dynamics code HOOMD-blue [3], tuning different system parameters and observing the variation in the thermodynam-ic crystal phases. We report a rich variety of self-assembled structures, ranging from the expected and well-known sphere packings and other simple structure types, to giant-unit cell structures and quasi-crystals [1]. The resulting phase diagrams and selected structures will be presented - some that are ubiquitous in atomic systems, as well as previously unknown ones.

[1] M. Engel, P. F. Damasceno, C. L. Phillips, S. C. Glotzer, Nature Mater. 14, 109-116 (2015).

[2] J. Dshemuchadse, D. Y. Jung, W. Steurer, Acta Crystallogr. B 67, 269-292 (2011).

[3] J. A. Anderson, S. C. Glotzer, http://arXiv.org/abs/1308.5587 (2013).
MS25-O2 Magnetic properties of Au-based Tsai-type approximants

\author{
Ryuji Tamura
}

1. Department of Materials Science and Technology, Tokyo University of Science

\section{email: tamura@rs.noda.tus.ac.jp}

The discovery of the icosahedral quasicrystals (QCs) [1] has shed light on the existences of closely-related crystals called "approximants", which are also made of the same icosahedral clusters found in QCs. Tsai-type approximants containing rare-earth $(\mathrm{R})$ icosahedra are of particular interest in view of their magnetic properties since they provide unique localized spin systems, i.e., periodic arrangement of $\mathrm{R}$ spin icosahedra. As a result of extensive investigations on a number of binary and ternary Tsai-type approximants, various magnetic orders including antiferromagnetic, ferromagnetic and spin-glass transitions have been observed and the origin of such different behaviours has now become an interesting issue. Moreover, recently, a composition-driven spin glass to ferromagnetic transition has been observed in Au-Al-Gd [2], which indicates that the magnetic order of approximants is governed by the oscillating nature of the RKKY interaction. This observation will give us a hint on how to tune the magnetic order of approximants as well as QCs. In this talk, we will give an overview of the magnetic transition phenomena in the approximants composed of $\mathrm{R}$ spin icosahedra and discuss the behaviours of spin icosahedra embedded in a bcc lattice, in terms of the RKKY interaction, local anisotropy and frustration. Recent new findings will be also presented.

[1] D. Shechtman, et al., Phys. Rev. Lett. 53, 1951 (1984).

[2] A. Ishikawa et al., Phys. Rev. B 93, 024416 (2016).

Keywords: quasicrystal, approximant, magnetism

Keywords: soft matter crystallography, complex structures, aperiodic structures, self-assembly 